Ind. Health, 1968, 6, 1.

\title{
EVALUATION METHODS FOR VIBRATION EFFECT
}

\author{
PART 4. MEASUREMENTS OF VIBRATION GREATNESS \\ FOR WHOLE BODY AND HAND IN VERTICAL \\ AND HORIZONTAL VIBRATIONS
}

Toshisuke MIWA

National Institute of Industrial Health, Kizuki-Sumiyoshi, Kawasaki

(Received May 1, 1968)

\begin{abstract}
A scale of vibration greatness (VG) corresponding to loudness (sone) in psychoacoustics was determined on the whole body and hand vibrations in vertical and horizontal directions. This scale has true ratio properties, and numbers on this scale having a given ratio refer to vibrations whose sensation has that ratio. The corrected ratio method devised by W. R. Garner for loudness measurement was used in our experiment. This method consists of two experiments which are based on fractionation and equisection judgments. The unit value of VG was decided as $40 \mathrm{VGL}(1 \mathrm{VG}=40$ VGL). Vibration greatness level (VGL) corresponds to loudness level (phon) as defined in Part 1. The experiments were carried out mainly at $20 \mathrm{c} / \mathrm{s}$. The effect of frequency on vibration greatness was examined at 5,30 and $60 \mathrm{c} / \mathrm{s}$. The vibration greatness for both of whole body and hand can be shown by following two equations.

$$
\begin{aligned}
& \log V G=0.030 \text { VGL-1.20, } \\
& \log V G=0.023 \text { VGL-0.92, } 1 \text { above } 1 \text { VG. }
\end{aligned}
$$

Dependency on frequency was not observed.
\end{abstract}

In Part 1, for rating the vibration in residential, industrial, and traffic areas with respect to their annoying qualities such as interference with rest, working efficiency and social activities, the frequency characteristics of the equal sensation for whole body vibration in vertical and horizontal directions were measured on the subjects at sitting and standing postures. The vibration greatness level (VGL) of any vibration corresponding to loudness level (phon) was defined as the vibration acceleration level (VAL) at the standard frequency of $20 \mathrm{c} / \mathrm{s}$ sensationally equalized to that vibration. The VAL was indicated as $20 \log _{10}\left(a / a_{\text {ref }}\right)$ where $a$; rms vibration acceleration level $(\mathrm{g})$ and $a_{\text {ref }} ; 10^{-3} \mathrm{~g}$.

In Part 3 , for evaluating hand vibration produced by portable vibrating tools such as rock drill, chain saw, air hammer and tytamper, the contours of vibration greatness level for hand were also obtained at similar working posture handling those tools.

From both VGL contours, the vibrations of various frequencies can be converted into equivalent vibration of $20 \mathrm{c} / \mathrm{s}$ selected as the standard in the measurement of VGL value. When a vibration has only one predominant 


\section{T. MIWA}

frequency component, the VGL scale will be effectively applied to the assessment of such kind of vibration. However, if a vibration has several frequency components with almost the same intensity in its spectrum or the uniformly continuous spectrum, the rating cannot be solved only by the VGL contours. Because the VGL scale is the interval scale, the sum of each VGL values of their frequency components does not correspond to the vibration sensation experienced actually. For example, when the vibration has two different frequency components, $30 \mathrm{VGL}$ and $40 \mathrm{VGL}$, the sum of VGL values $(30+40=70$ VGL) does not mean the equal sensation for the compound vibration of both components.

The same problem had also been experienced in the acoustics especially for the assessment of noise. To solve this problem, for the first step, the loudness scale (sone) which has true ratio property has been proposed after the various experimental investigations.

Among the many techniques developed to obtain the loudness scale in psycho-acoustics, the method of corrected ratios devised by W. R. Garner1) seems to be rational and it may be very useful for constructing the vibration greatness scale corresponding to loudness. This method consists of two separate experiments of fractionation judgment and equisection judgment. In the former, the observers are required to adjust the intensity of one tone to be of given ratio to loudness of another fixed tone. While, in the latter, the observers are asked to adjust a series of tones between two fixed end tones for producing equal interval in loudness. For example, the difference in loudness between the lowest tone and the next lowest is the same as the difference between the highest and the next highest, etc.

The vibration greatness scale for whole body vibration in vertical and horizontal directions at sitting postures and for the hand vibration in vertical direction are reported in this paper.

\section{Procedures}

\section{The fractionation experiment}

In the corrected ratio method, the first experiment based on the fractionation judgment will allow the construction of a VG scale with a verbalized ratio instead of the true ratio and with a true zero point. This scale is indicated as

$$
\text { VIBRATION GREATNESS }=\alpha \cdot \beta^{n}
$$

$\alpha$; an arbitrary constant to shift numbers on the VG scale to the proper value, e. g. $1 \mathrm{VG}$ in this scale is defined as $40 \mathrm{VGL}, \boldsymbol{\beta}$; the fractionation ratio which means the verbalized and does not mean the true ratio value, $n$; the successive ratio $(0,-1,-2,-3, \ldots .$.$) . For example, when a vibration is equalized in$ sensation to its one-half value, the verbalized ratio $(\beta)$ becomes 2 .

Ten male subjects were asked to describe the vibration level which would 


\section{EVALUATION METHODS FOR VIBRATION EFFECT (4)}

be felt as one-half in comparison with the standard level. The measurement was done by the method of paired comparisons. The subjects received the standard vibration, then received the variable vibration and pause for 4 sec for each. This series, composed of two kinds of vibration and pause, was repeated changing only the level of the variable vibration until the subjects would find out the sensation point to be the half of the standard. The level of the variable vibration was controlled by the tester with the descending and ascending series. In descending series, the variable vibration level was begun from the same level as the standard. On the other hand, in the ascending series the variable vibration was started from the level which was lower by $10 \mathrm{~dB}$ than the level determined as the one-half sensation value in the descending series. This measurement was tried at five or six VGL values between 17 and 67, for example, at $20 \mathrm{c} / \mathrm{s}, 17,27,37,47$, and $57 \mathrm{VGL}$ for whole body vertical vibration, $27,37,47,57$ and 67 VGL for whole body horizontal vibration, and $37,43,51,57,63$ and 67 VGL for hand vibration.

The obtained data were plotted on the graph whose abscissa represented the VGL value and ordinate the value judged by the subject as one-half level. At the same time, the straight line with $45^{\circ}$ slope was drawn on this figure. The point on this line corresponding to the maximum VGL value examined now was selected as $n=0$. From this point $(n=0)$, a perpendicular was let fall to the observed one-half sensation curve and the intersecting point on this curve was defined as $n=-1$. Then, when the line drawing parallel to the abscissa from the point $n=-1$ intersects the $45^{\circ}$ line, from this intersecting point, the second perpendicular was drawn to the observed curve. The intersecting point on this curve was called $n=-2$ and so on down the VGL value continuum. Lastly, the relationship between the successive ratios and the judged VGL values would be drawn.

\section{The equisection experiment}

The second measurement based on the equisection judgments will permit the construction of a scale of the vibration greatness with true interval natures but with an unknown intercept constant. This relation is denoted as

$$
\text { VIBRATION GREATNESS }=\boldsymbol{r}(\boldsymbol{T}-\boldsymbol{\delta})
$$

provided that $T$ is a VG value with an arbitrary criterion point, and $\delta$ an intercept constant correcting discretion of the criterion point of $T$, and $\gamma$ the same kind of arbitrary constant as $\alpha$ in equation (1).

The subjects were requested to sensationally determine three points being at equal intervals between the highest and the lowest end of vibration levels. The range between 17-67 VGL was divided into three overlapping parts because of easiness of subjective judgment and of improvement of reliability of observed values. For example, the divided parts at $20 \mathrm{c} / \mathrm{s}$ were chosen as 17-37, 27-47 and 37-57 VGL for whole body vertical vibration, 27-47, 37-57 and 47-67 VGL 


\section{T. MIWA}

for whole body horizontal vibration, and 37-53, 45-63 and 53-67 VGL for hand vibration. In each part, the subjects first bisected the total interval and then next bisections were tried for each bisected part. Lastly, these three judged levels were given successively to the subjects to check the correctness of their judgments. The measurement was carried out by the method of paired comparisons. The subject received the first fixed vibration, the variable vibration, the second fixed one and pause successively. The levels of the first and the second fixed vibrations were the upper and the lower end levels respectively in descending series, while in ascending, they were conversely the lower and the upper end levels. This series was repeated until the judgment would be established. The level of the variable vibration was controlled by the tester in descending and ascending order. In descending series, the variable vibration was started from the same level as the fixed one of the upper end. While, in ascending series, the variable vibration was begun from the same level as the fixed one of the lower end.

In order to put all the values observed in three divided ranges to one smoothed curve, proportional allotment was used. This curve shows the relations between the VG value with arbitrary zero point and the VGL value.

\section{Determination of $\delta$ and $\beta$}

The following relation is derived from the logarithmic form of Eqs. 1 and 2,

$$
\log (T-\delta)=(\log \beta) \cdot n+C
$$

where $C=\log (\alpha / \gamma)$. This equation means that the logarithmic value of the measured $T$ can indicate linearity to $n$ by putting arbitrary values into $\delta$ by the method of trial and error. The slope of this line (Eq. 3) corresponds to $\log \beta$, and the values of $\alpha$ and $\gamma$ are fixed on account of the operation to make 1 VG agree with 40 VGL.

\section{The apparatus}

The apparatus of the experiment were composed of the oscillator, five attenuators, power amplifier and the vibration table of the electro-dynamic type

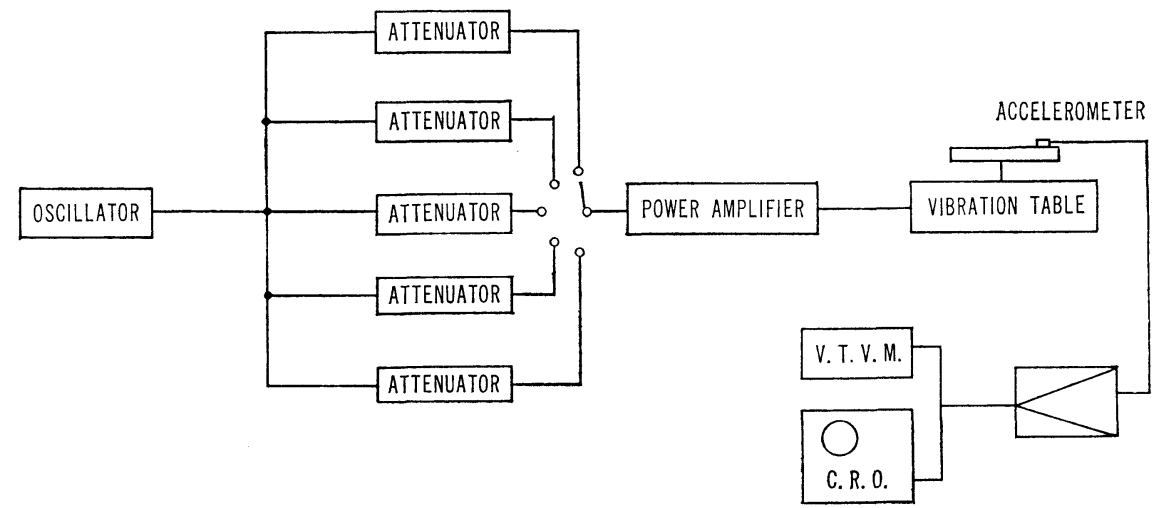

Fig. 1. The block diagram of the experimental apparatus. 
described in the previous parts. The block diagram of this experiment is shown in Fig. 1. The experiment was mainly done at $20 \mathrm{c} / \mathrm{s}$, because the standard frequency in the equal sensation experiment had previously been selected as $20 \mathrm{c} / \mathrm{s}$. To check the frequency dependence of the vibration greatness scale, the same experiments were carried out at 5 and $60 \mathrm{c} / \mathrm{s}$ for the whole body in both directions, and at 30 and $60 \mathrm{c} / \mathrm{s}$ for the hand.

\section{Postures of subjects}

The measurements of the whole body vibrations in the vertical and the horizontal directions were tried on the subjects sitting erect alone, because in the preliminary experiment studied on three subjects the result for the standing erect was almost the same as that for the sitting erect.

For the hand vibration, the vertical direction was only tested, as it had been known in Part 3 that the vibration sensation of the hand did not show the difference with the vibration directions. The subject pressed by hand the vibration table of the aluminum disc by about $5 \mathrm{kgw}$. This compressing force was regulated by watching the strain meter of which the strain gages were applied on the upper leaf spring of the vibration table of small electro-dynamic type. The joints of hand, elbow and shoulder were bent at right angle in order to simulate the working posture handling the portable vibrating tools and, in addition, not to transmit the vibration to head.

\section{Results AND Discussions}

In derivation of the vibration greatness scale the following items were examined on every case of the whole body and the hand vibrations. To simplify the description, these items are discussed by the instances obtained for the whole body vertical vibration at $20 \mathrm{c} / \mathrm{s}$ alone.

(1) Verification for the correctness of Eq. 3, constructed on hearing, to the vibration sensation. (2) Difference of two VG scales calculated by different methods. The one is determined from averaging ten individual VG scales observed with each person (individual data), the other is calculated from the raw data pooled with 10 subjects (pooled data). (3) Differences between the VG scales obtained from the corrected ratio method, the fractionation method uncorrected on $\beta$ and the equisection method uncorrected on $\delta$. (4) The correlation of the VG scales derived from the fractionation method corrected on $\beta$ and the equisection method corrected on $\delta$.

To prove the correctness of Eq. 3, the observed values of $\log (\boldsymbol{T}-\boldsymbol{\delta})$ and $n$ with 10 subjects are plotted in Fig. 2 in which, on the case uncorrected on $\delta$ and $\beta$, there indicates no linear relation between them as shown in the Figure. After the correcting calculation described previously, the linearity between $\log (T-\delta)$ and $n$ can be found, and $\delta$ and $\beta$ were determined as 3.40 and 1.71 


\section{T. MIWA}

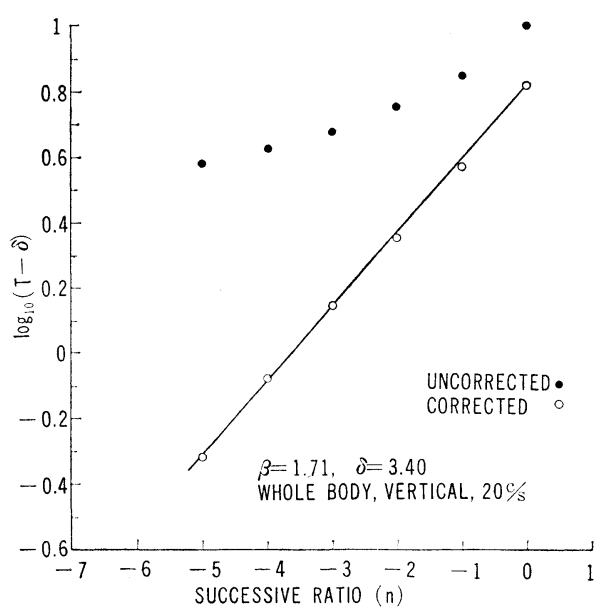

Fig. 2. The relation of $\log (T-\delta)$ and $n$ through VGL as a parameter for the vertical vibration of whole body at $20 \mathrm{c} / \mathrm{s}$ with 10 observers. The solid circle (O) shows the uncorrected case $(\delta=0)$ and the circle $(\bigcirc)$ the corrected $(\delta=3.40)$. From the slope of this line, the value of $\beta$ is determined as 1.71 .

respectively. This fact shows that Eq. 3 holds good for vibration sensation. In the other cases of the horizontal whole body and the hand vibrations, this fact has been proved similarly.

The individual and pooled data functions on the VG scale were in good agreement with each other except lower VGL value as shown in Fig. 3. The difference in the lower VGL part is considered to be negligibly small for the purpose of evaluation of vibration. This fact was quite similar to the other

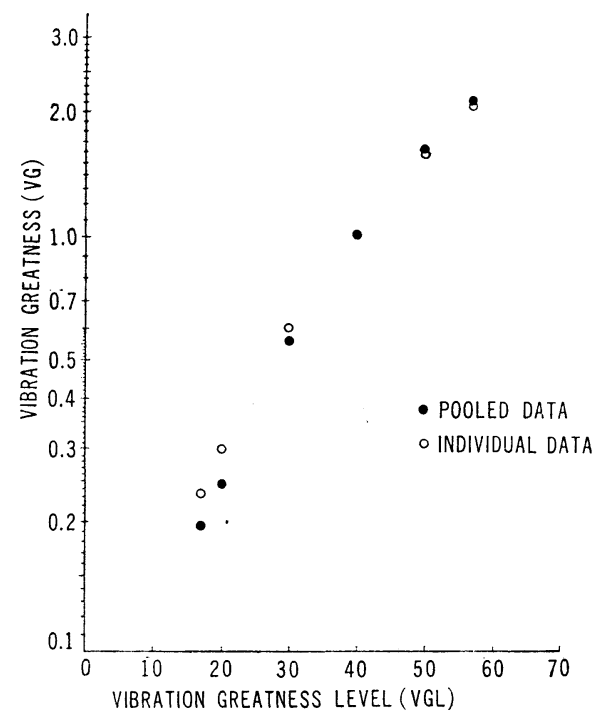

Fig. 3. The difference between the individual and the pooled data on the relation of VG-VGL for the vertical vibration of whole body at $20 \mathrm{c} / \mathrm{s}$ with 10 subjects. 


\section{EVALUATION METHODS FOR VIBRATION FFFECT (4)}

cases of the horizontal whole body and the hand vibrations. About both functions on the VG scale, the pooled data function may be desirable, because the calculation procedure is simpler than the individual data function.

Variability of the VG scale with individual data, and differences between the three VG functions (pooled data functions obtained from the corrected ratio method, from the fractionation method uncorrected with $\beta$ and from the equisection method uncorrected with $\delta$ ) are shown in Fig. 4 a and $\mathrm{b}$ respectively. The dispersion on lower VGL value was considerably larger because of difficulty of judgment as seen in Fig. 4, a. It may be known that the VG scale obtained from the corrected ratio method indicates approximately average values of those from the uncorrected fractionation and uncorrected equisection methods as shown in Fig. 4, b.

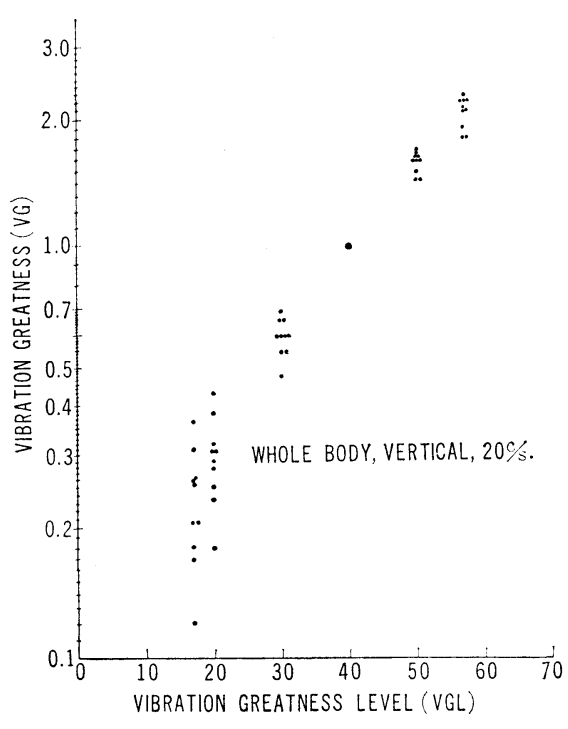

(a)

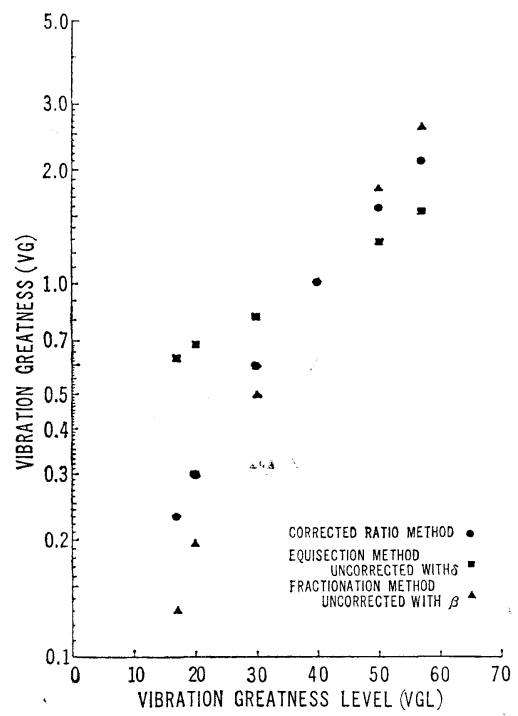

(b)

Fig. 4, a. Dispersions of the individual data obtained from the corrected ratio method for the vertical vibration of whole body at $20 \mathrm{c} / \mathrm{s}$ with 10 subjects.

Fig. 4, b. Comparison of three results calculated with the pooled data by the corrected ratio method, the equisection method uncorrected with $\delta$ and the fractionation method uncorrected with $\beta$.

The individual VG functions obtained from the fractionation and the equisection methods corrected with $\beta$ and $\delta$ respectively are plotted in Fig. 5 in order to examine the correlation of both. This figure shows the good correlation with each other. This tendency was also observed in the other vibration cases. Therefore, the average operation with the data obtained from both methods is known not to lose physical meaning. 


\section{T. MIWA}

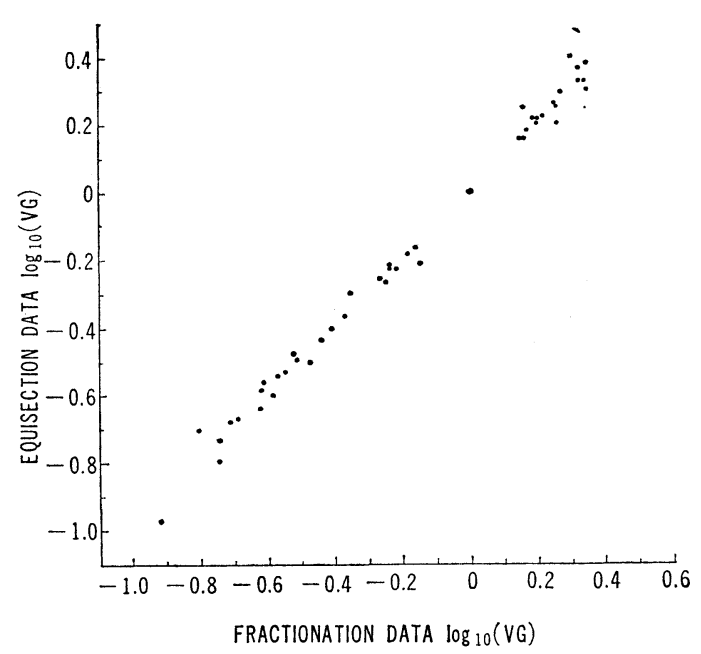

Fig. 5. Correlation of two VG scales with individual data obtained from the fractionation method and the equisection method corrected on $\beta$ and $\delta$ respectively. The individual data were measured for the whole body vertical vibration at $20 \mathrm{c} / \mathrm{s}$ on 10 subjects.

In acoustics, S. S. Stevens ${ }^{2)}$ described mistrust of the equisection experiment on the two points. The one was occurence of rather large differences of loudness depending upon the order by which the tones were presented in the ascending or descending series. The other was the question whether the subject was able to bisect an interval without being influenced by the ratios as well as the differences between the loudnesses. In vibration experiments, these questions were also thought, but the difference of VG scale for vibration presentation order was not observed so clearly in our experiment. The other doubt of interval bisection of $\mathrm{S}$. S. Stevens may not be considered to be essential, because the dispersion of the VG scale with the individual data obtained from the equisection method was not so large. If an individual subject understands meaning of the equisection in different semantics, the dispersion of the data will be expected to extend. Moreover, as psychological scales always can not be derived uniquely as mathematical theory, the results are desirable to be corrected by values of the same kinds which are obtained by the other method based on the different hypothesis. In addition, there was observed the good correlation between fractionation and equisection method corrected on $\beta$ and $\delta$ respectively with individual data. Therefore, the corrected ratio method was adopted in this report.

Consequently, all the result on the whole body and hand vibrations obtained by the corrected ratio method with the pooled data are plotted in Fig. 6 .

From this graph, the vibration greatness may be estimated by one curve.

In order to compare the VG scale with loudness functions observed by W. R. Garner and S. S. Stevens, these functions are superposed on the graph of VG 


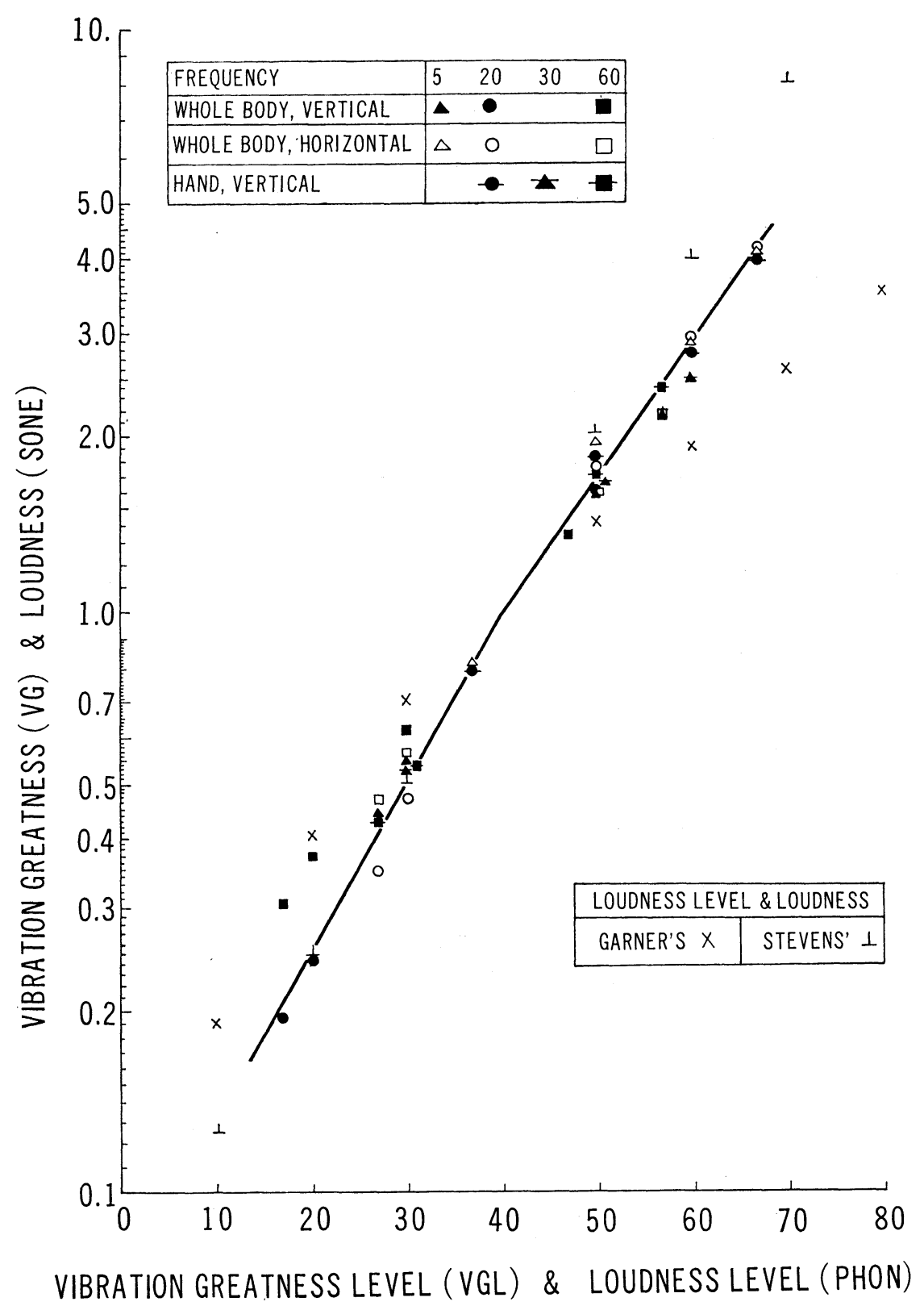

Fig. 6. The relations of VG-VGL at $20 \mathrm{c} / \mathrm{s}$ on the whole body and the hand vibration and their frequency dependence at 5,30 and $60 \mathrm{c} / \mathrm{s}$. The relations between loudness level and loudness in psycho-acoustics obtained by W. R. Garner and S. S. Stevens are also shown in this graph. The unit of VG has been taken as $40 \mathrm{VGL}(1 \mathrm{VG}=40 \mathrm{VGL})$. 


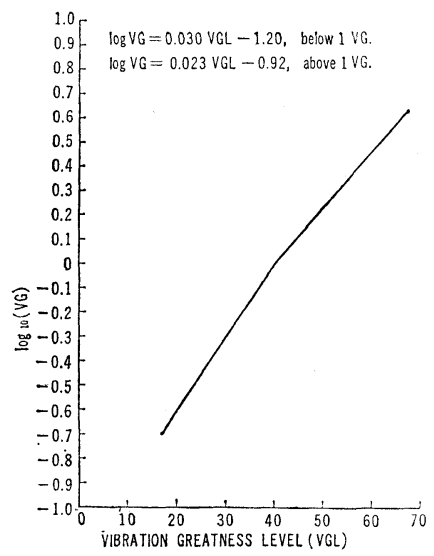

Fig. 7. The relation of $\log$ VG and VGL.

values as denoted in Fig. 6. The vibration greatness function is found to show average values between these two loudness functions in the range above $1 \mathrm{VG}$ and to agree with the curve of S. S. Stevens below 1 VG.

For checking the frequency dependence of the VG scale, the pooled data functions on 10 subjects obtained at 5 and $60 \mathrm{c} / \mathrm{s}$ for whole body vertical and horizontal vibrations and at 30 and $60 \mathrm{c} / \mathrm{s}$ for the hand vibration are also plotted on the same graph of Fig. 6. The dependence of the VG scale on frequency was not observed as shown in this figure.

Lastly, the curve of VG and VGL is redrawn on the graph of log VG and VGL in Fig. 7 and this relation may be formulated in the following equations,

$$
\begin{aligned}
& \log \mathrm{VG}=0.030 \mathrm{VGL}-1.20, \\
& \log \mathrm{VG}=0.023 \mathrm{VGL}-0.92, \text { above } 1 \mathrm{VG} .
\end{aligned}
$$

So, mutual conversion between VG and VGL can be obtained by these equations.

\section{REFERENCES}

1) Garner W. R. (1954). J. Acoust. Soc. Am., 26. 73.

2) Stevens S. S. (1955). J. Acoust. Soc. Am., 27. 815. 